\title{
A SUZUKI TYPE COMMON TRIPLED FIXED POINT THEOREM FOR A HYBRID PAIR OF MAPPINGS
}

\author{
STOJAN RADENOVIĆ, K. P. R. RAO, K. V. SIVA PARVATHI, \\ AND TATJANA DOŠENOVIĆ
}

Received 26 October, 2014

\begin{abstract}
In this paper, we prove a tripled common fixed point theorem of Suzuki type for a pair of hybrid mappings in metric spaces. Our result generalizes and modifies several comparable results in the literature. We also provide an example to support our theorem.
\end{abstract}

2010 Mathematics Subject Classification: 47H10; 54H25

Keywords: Hausdorff-Pompeu metric, multi-valued mapping, tripled fixed point, w-compatible mappings

\section{INTRODUCTION AND PRELIMINARIES}

Banach contraction principle plays a very important role in nonlinear analysis and has many generalizations. In 2008, Suzuki $[37,38]$ proved generalized versions of both Banach's and Edelstein's basic results. Many other works in this direction have been considered, for example $[2,4,13,15,19,20,23,25,29,35,36]$ and the references therein.

The study of fixed points for multivalued contraction mappings using the HausdorffPompeu metric was initiated by Nadler [28]. Let $(X, d)$ be a metric space. We denote $C B(X)$ the family of all nonempty closed and bounded subsets of $X$ and $C L(X)$ the set of all nonempty closed subsets of $X$. For $A, B \in C B(X)$ and $x \in X$, we denote $D(x, A)=\inf \{d(x, a): a \in A\}$. Let $H$ be the Hausdorff-Pompeu metric induced by the metric $d$ on $X$, that is

$$
H(A, B)=\max \left\{\sup _{x \in A} d(x, B), \sup _{y \in B} d(y, A)\right\}
$$

for every $A, B \in C B(X)$.

It is clear that for $A, B \in C B(X)$ and $a \in A$ we have $d(a, B) \leq H(A, B)$.

Definition 1. An element $x \in X$ is said to be a fixed point of a multivalued mapping $T: X \rightarrow C B(X)$ if and only if $x \in T x$.

The fourth author was supported by the project MNTRRS-174009. 
In 1969, Nadler [28] extended the famous Banach contraction principle [10] from singlevalued mapping to multivalued mapping and proved the following fixed point theorem for the multivalued contraction.

Theorem 1 (Nadler, [28]). Let $(X, d)$ be a complete metric space and let $T$ be a mapping from $X$ into $C B(X)$. Assume that there exists $c \in[0,1)$ such that

$$
H(T x, T y) \leq c d(x, y),
$$

for all $x, y \in X$. Then, $T$ has a fixed point.

Lemma 1 (Nadler, [28]). Let $A, B \in C B(X)$ and $h>1$. Then for every $a \in A$, there exists $b \in B$ such that $d(a, b) \leq h H(A, B)$.

The existence of fixed points for various multivalued contractive mappings has been studied by many authors under different conditions. For details, we refer the reader to $[3,16-18,21,24,26,33]$ and the references therein.

In 2011, Samet and Vetro [34] introduced the concept of coupled fixed point for multivalued mapping. Very recently, Berinde and Borcut [11,12] proved some tripled fixed and coincidence point theorems for contractive type mappings in partially ordered metric spaces. Later, several authors obtained coincidence and common tripled fixed point theorems in various spaces, for example refer to $[1,5-9,14$, 27, 30-32].

The aim of this paper is to combine the ideas of tripled fixed points and Suzuki type fixed point theorems to obtain a tripled common fixed point theorem for a pair of hybrid mappings in a metric space.

First, we give the following theorem of Suzuki [37].

Theorem 2 ([37]). Let $(X, d)$ be a complete metric space and let $T$ be a mapping on $X$. Define a nonincreasing function $\theta$ from $[0,1)$ into $\left(\frac{1}{2}, 1\right]$ by

$$
\theta(r)= \begin{cases}1, & 0 \leq \mathrm{r} \leq \frac{\sqrt{5}-1}{2} \\ \frac{1-r}{r^{2}}, & \frac{\sqrt{5}-1}{2} \leq \mathrm{r} \leq \frac{1}{\sqrt{2}} \\ \frac{1}{1+r}, & \frac{1}{\sqrt{2}} \leq \mathrm{r}<1 .\end{cases}
$$

Assume that $r \in[0,1)$, such that

$$
\theta(r) d(x, T x) \leq d(x, y) \text { implies } d(T x, T y) \leq r d(x, y) .
$$

for all $x, y \in X$. Then, there exists a unique fixed point $z$ of $T$. Moreover, $\lim _{n \rightarrow \infty} T^{n} x=$ $z$ for all $x \in X$.

Now, we give some known definitions which are used to prove our main result.

Definition 2 ([34]). An element $(x, y) \in X \times X$ is called a coupled fixed point of the mapping $F: X \times X \rightarrow C B(X)$ if $x \in F(x, y)$ and $y \in F(y, x)$. 
Definition 3 ([22]). An element $(x, y) \in X \times X$ is called

(i) a coupled coincident point of mappings $F: X \times X \rightarrow C B(X)$ and $g: X \rightarrow X$ if $g x \in F(x, y)$ and $g y \in F(y, x)$;

(ii) a coupled common fixed point of mappings $F: X \times X \rightarrow X$ and $g: X \rightarrow X$ if $x=g x \in F(x, y)$ and $y=g y \in F(y, x)$.

Definition 4 ([31]). Let $X$ be a nonempty set, $F: X \times X \times X \rightarrow C B(X)$ and $g: X \rightarrow X$. Then point $(x, y, z) \in X \times X \times X$ is called a tripled

(i) fixed point of $F$ if $x \in F(x, y, z), y \in F(y, z, x)$ and $z \in F(z, x, y)$.

(ii) coincidence point of $F$ and $g$ if $g x \in F(x, y, z), g y \in F(y, z, x)$ and $g z \in$ $F(z, x, y)$.

(iii) common fixed point of $F$ and $g$ if $x=g x \in F(x, y, z), y=g y \in F(y, z, x)$ and $z=g z \in F(z, x, y)$.

Definition 5 ([31]). Let $X$ be a nonempty set, $F: X \times X \times X \rightarrow C B(X)$ and $g$ : $X \rightarrow X$. The pair $(F, g)$ is called $w$-compatible if $g(F(x, y, z)) \subseteq F(g x, g y, g z)$, whenever $(x, y, z)$ is a coincidence point of $F$ and $g$.

In this paper, we use the following function introduced in [20].

Let $\phi:[0,1) \rightarrow(0,1]$ be a nonincreasing function defined by

$$
\phi(\theta)=\left\{\begin{array}{cl}
1, & \text { if } 0 \leq \theta<\frac{1}{2} \\
1-\theta, & \text { if } \frac{1}{2} \leq \theta<1
\end{array}\right.
$$

Using the notion of w-compatibility of the pair $(F, g)$, we establish a tripled common fixed point theorem of Suzuki type for a hybrid pair of mappings in metric spaces. We support our result by an example.

\section{MAIN RESULTS}

Our main result is the following common tripled fixed point theorem.

Theorem 3. Let $(X, d)$ be a metric space, $F: X \times X \times X \rightarrow C B(X)$ and $g: X \rightarrow$ $X$ be mappings satisfying the following :

(i) $F(X \times X \times X) \subseteq g(X)$ and $g(X)$ is complete

(ii) If there exists $\theta \in[0,1)$ such that

$$
\phi(\theta) \min \left\{\begin{array}{c}
d(g x, F(x, y, z)), \\
d(g y, F(y, z, x)), \\
d(g z, F(z, x, y))
\end{array}\right\} \leq \max \left\{\begin{array}{c}
d(g x, g u), \\
d(g y, g v), \\
d(g z, g w)
\end{array}\right\}
$$


implies

$$
\begin{aligned}
& H(F(x, y, z), F(u, v, w)) \\
& \leq \theta \max \left\{\begin{array}{c}
d(g x, g u), d(g y, g v), d(g z, g w), \\
d(g x, F(x, y, z)), d(g y, F(y, z, x)), d(g z, F(z, x, y)), \\
d(g u, F(u, v, w)), d(g v, F(v, w, u)), d(g w, F(w, u, v)), \\
\frac{1}{2} \max \left\{\begin{array}{c}
d(g x, F(u, v, w)), d(g y, F(v, w, u)), d(g z, F(w, u, v)), \\
d(g u, F(x, y, z)), d(g v, F(y, z, x)), d(g w, F(z, x, y))
\end{array}\right\}
\end{array}\right\} .
\end{aligned}
$$

for all $x, y, z, u, v, w \in X$. Then $F$ and $g$ have a tripled coincidence point in $X \times$ $X \times X$.

Further, $F$ and $g$ have a tripled common fixed point if one of the following conditions holds:

(a) Suppose the pair $(F, g)$ is $w$-compatible and there exist $u, v, w \in X$ such that $\lim _{n \rightarrow \infty} g^{n} x=u, \lim _{n \rightarrow \infty} g^{n} y=v$ and $\lim _{n \rightarrow \infty} g^{n} z=w$, whenever $(x, y, z)$ is a tripled coincidence point of $F$ and $g$ and $g$ is continuous at $u, v$ and $w$.

(b) Suppose there exist $u, v, w \in X$ such that $\lim _{n \rightarrow \infty} g^{n} u=x, \lim _{n \rightarrow \infty} g^{n} v=y$ and $\lim _{n \rightarrow \infty} g^{n} w=z$, whenever $(x, y, z)$ is a tripled coincidence point of $F$ and $g$ and $g$ is continuous at $x, y$ and $z$.

Proof. Let $x_{0}, y_{0}, z_{0} \in X$. From (i) there exist $x_{1}, y_{1}, z_{1} \in X$ such that $g x_{1} \in$ $F\left(x_{0}, y_{0}, z_{0}\right), g y_{1} \in F\left(y_{0}, z_{0}, x_{0}\right)$ and $g z_{1} \in F\left(z_{0}, x_{0}, y_{0}\right)$. By Lemma 1 , there exist $x_{2}, y_{2}, z_{2} \in X$ such that

$$
\begin{aligned}
& g x_{2} \in F\left(x_{1}, y_{1}, z_{1}\right) \text { with } d\left(g x_{1}, g x_{2}\right) \leq \frac{1}{\sqrt{\theta}} H\left(F\left(x_{0}, y_{0}, z_{0}\right), F\left(x_{1}, y_{1}, z_{1}\right)\right), \\
& g y_{2} \in F\left(y_{1}, z_{1}, x_{1}\right) \text { with } d\left(g y_{1}, g y_{2}\right) \leq \frac{1}{\sqrt{\theta}} H\left(F\left(y_{0}, z_{0}, x_{0}\right), F\left(y_{1}, z_{1}, x_{1}\right)\right), \\
& g z_{2} \in F\left(z_{1}, x_{1}, y_{1}\right) \text { with } d\left(g z_{1}, g z_{2}\right) \leq \frac{1}{\sqrt{\theta}} H\left(F\left(z_{0}, x_{0}, y_{0}\right), F\left(z_{1}, x_{1}, y_{1}\right)\right) .
\end{aligned}
$$

Continuing in this way, we get sequences $\left\{x_{n}\right\},\left\{y_{n}\right\}$ and $\left\{z_{n}\right\}$ in $X$ such that $g x_{n+1} \in F\left(x_{n}, y_{n}, z_{n}\right), g y_{n+1} \in F\left(y_{n}, z_{n}, x_{n}\right)$ and $g z_{n+1} \in F\left(z_{n}, x_{n}, y_{n}\right)$ with

$$
\begin{aligned}
& d\left(g x_{n}, g x_{n+1}\right) \leq \frac{1}{\sqrt{\theta}} H\left(F\left(x_{n-1}, y_{n-1}, z_{n-1}\right), F\left(x_{n}, y_{n}, z_{n}\right)\right), \\
& d\left(g y_{n}, g y_{n+1}\right) \leq \frac{1}{\sqrt{\theta}} H\left(F\left(y_{n-1}, z_{n-1}, x_{n-1}\right), F\left(y_{n}, z_{n}, x_{n}\right)\right), \\
& d\left(g z_{n}, g z_{n+1}\right) \leq \frac{1}{\sqrt{\theta}} H\left(F\left(z_{n-1}, x_{n-1}, y_{n-1}\right), F\left(z_{n}, x_{n}, y_{n}\right)\right) .
\end{aligned}
$$

Case i: Suppose $g x_{n}=g x_{n+1}, g y_{n}=g y_{n+1}$ and $g z_{n}=g z_{n+1}$, for some $n$. Then $g x_{n} \in F\left(x_{n}, y_{n}, z_{n}\right), g y_{n} \in F\left(y_{n}, z_{n}, x_{n}\right)$ and $g z_{n} \in F\left(z_{n}, x_{n}, y_{n}\right)$. Thus $\left(x_{n}, y_{n}, z_{n}\right)$ is a tripled coincidence point of $F$ and $g$. 
Case ii: Assume that $g x_{n} \neq g x_{n+1}$ or $g y_{n} \neq g y_{n+1}$ or $g z_{n} \neq g z_{n+1}$ for all $n$. Since

$$
\phi(\theta) \min \left\{\begin{array}{c}
d\left(g x_{0}, F\left(x_{0}, y_{0}, z_{0}\right)\right), \\
d\left(g y_{0}, F\left(y_{0}, z_{0}, x_{0}\right)\right), \\
d\left(g z_{0}, F\left(z_{0}, x_{0}, y_{0}\right)\right)
\end{array}\right\} \leq \max \left\{\begin{array}{c}
d\left(g x_{0}, g x_{1}\right), \\
d\left(g y_{0}, g y_{1}\right), \\
d\left(g z_{0}, g z_{1}\right)
\end{array}\right\},
$$

from (2.2), we have

$$
\leq \theta \max \left\{\begin{array}{c}
d\left(F\left(x_{0}, y_{0}, z_{0}\right), F\left(x_{1}, y_{1}, z_{1}\right)\right) \\
d\left(g x_{0}, F\left(x_{0}, y_{0}, z_{0}\right)\right), d\left(g y_{0}, F\left(y_{0}, z_{0}, x_{0}\right)\right), d\left(g z_{0}, F\left(z_{0}, x_{0}, y_{0}\right)\right), \\
d\left(g x_{1}, F\left(x_{1}, y_{1}, z_{1}\right)\right), d\left(g y_{1}, F\left(y_{1}, z_{1}, x_{1}\right)\right), d\left(g z_{1}, F\left(z_{1}, x_{1}, y_{1}\right)\right), \\
\frac{1}{2} \max \left\{\begin{array}{c}
\left.d\left(g x_{0}, F\left(x_{1}, y_{1}, z_{1}\right)\right)\right) d\left(g y_{0}, F\left(y_{1}, z_{1}, x_{1}\right)\right), d\left(g z_{0}, F\left(z_{1}, x_{1}, y_{1}\right)\right), \\
d\left(g x_{1}, F\left(x_{0}, y_{0}, z_{0}\right)\right), d\left(g y_{1}, F\left(y_{0}, z_{0}, x_{0}\right)\right), d\left(g z_{1}, F\left(z_{0}, x_{0}, y_{0}\right)\right)
\end{array}\right\}
\end{array}\right\} .
$$

Now we have, using triangular inequality

$$
d\left(g x_{1}, g x_{2}\right) \leq \frac{1}{\sqrt{\theta}} H\left(F\left(x_{0}, y_{0}, z_{0}\right), F\left(x_{1}, y_{1}, z_{1}\right)\right) \leq \sqrt{\theta} \max \left\{\begin{array}{c}
d\left(g x_{0}, g x_{1}\right), d\left(g y_{0}, g y_{1}\right), \\
d\left(g z_{0}, g z_{1}\right), d\left(g x_{1}, g x_{2}\right), \\
d\left(g y_{1}, g y_{2}\right), d\left(g z_{1}, g z_{2}\right)
\end{array}\right\} .
$$

Similarly, we have

$$
d\left(g y_{1}, g y_{2}\right) \leq \sqrt{\theta} \max \left\{\begin{array}{c}
d\left(g x_{0}, g x_{1}\right), d\left(g y_{0}, g y_{1}\right) \\
d\left(g z_{0}, g z_{1}\right), d\left(g x_{1}, g x_{2}\right) \\
d\left(g y_{1}, g y_{2}\right), d\left(g z_{1}, g z_{2}\right)
\end{array}\right\}
$$

and

$$
d\left(g z_{1}, g z_{2}\right) \leq \sqrt{\theta} \max \left\{\begin{array}{c}
d\left(g x_{0}, g x_{1}\right), d\left(g y_{0}, g y_{1}\right) \\
d\left(g z_{0}, g z_{1}\right), d\left(g x_{1}, g x_{2}\right) \\
d\left(g y_{1}, g y_{2}\right), d\left(g z_{1}, g z_{2}\right)
\end{array}\right\}
$$

Thus

$$
\max \left\{\begin{array}{c}
d\left(g x_{1}, g x_{2}\right), \\
d\left(g y_{1}, g y_{2}\right), \\
d\left(g z_{1}, g z_{2}\right)
\end{array}\right\} \leq \sqrt{\theta} \max \left\{\begin{array}{c}
d\left(g x_{0}, g x_{1}\right), d\left(g y_{0}, g y_{1}\right), \\
d\left(g z_{0}, g z_{1}\right), d\left(g x_{1}, g x_{2}\right), \\
d\left(g y_{1}, g y_{2}\right), d\left(g z_{1}, g z_{2}\right)
\end{array}\right\} .
$$

If

$$
\max \left\{\begin{array}{c}
d\left(g x_{0}, g x_{1}\right), d\left(g y_{0}, g y_{1}\right) \\
d\left(g z_{0}, g z_{1}\right), d\left(g x_{1}, g x_{2}\right) \\
d\left(g y_{1}, g y_{2}\right), d\left(g z_{1}, g z_{2}\right)
\end{array}\right\}=d\left(g x_{1}, g x_{2}\right),
$$

then from (2.3) we have $g x_{1}=g x_{2}$.

Analogous other two cases

$$
\max \left\{\begin{array}{c}
d\left(g x_{0}, g x_{1}\right), d\left(g y_{0}, g y_{1}\right), \\
d\left(g z_{0}, g z_{1}\right), d\left(g x_{1}, g x_{2}\right), \\
d\left(g y_{1}, g y_{2}\right), d\left(g z_{1}, g z_{2}\right)
\end{array}\right\}=d\left(g y_{1}, g y_{2}\right),
$$


and

$$
\max \left\{\begin{array}{c}
d\left(g x_{0}, g x_{1}\right), d\left(g y_{0}, g y_{1}\right) \\
d\left(g z_{0}, g z_{1}\right), d\left(g x_{1}, g x_{2}\right) \\
d\left(g y_{1}, g y_{2}\right), d\left(g z_{1}, g z_{2}\right)
\end{array}\right\}=d\left(g z_{1}, g z_{2}\right)
$$

imply that $g y_{1}=g y_{2}$ and $g z_{1}=g z_{2}$. It is a contradiction. Hence from (2.3), we have

$$
\max \left\{\begin{array}{c}
d\left(g x_{1}, g x_{2}\right), \\
d\left(g y_{1}, g y_{2}\right), \\
d\left(g z_{1}, g z_{2}\right)
\end{array}\right\} \leq \sqrt{\theta} \max \left\{\begin{array}{c}
d\left(g x_{0}, g x_{1}\right), d\left(g y_{0}, g y_{1}\right), \\
d\left(g z_{0}, g z_{1}\right)
\end{array}\right\}
$$

Continuing in this way, we get

$$
\max \left\{\begin{array}{c}
d\left(g x_{n}, g x_{n+1}\right), \\
d\left(g y_{n}, g y_{n+1}\right), \\
d\left(g z_{n}, g z_{n+1}\right)
\end{array}\right\} \leq(\sqrt{\theta})^{n} \max \left\{\begin{array}{c}
d\left(g x_{0}, g x_{1}\right), d\left(g y_{0}, g y_{1}\right), \\
d\left(g z_{0}, g z_{1}\right)
\end{array}\right\},
$$

which in turn yields that

$$
\max \left\{\begin{array}{c}
\sum d\left(g x_{n}, g x_{n+1}\right), \\
\sum d\left(g y_{n}, g y_{n+1}\right), \\
\sum d\left(g z_{n}, g z_{n+1}\right)
\end{array}\right\} \leq \sum(\sqrt{\theta})^{n} \max \left\{\begin{array}{c}
d\left(g x_{0}, g x_{1}\right), d\left(g y_{0}, g y_{1}\right), \\
d\left(g z_{0}, g z_{1}\right)
\end{array}\right\}<\infty
$$

Hence $\left\{g x_{n}\right\},\left\{g y_{n}\right\}$ and $\left\{g z_{n}\right\}$ are Cauchy sequences. Since $g(X)$ is complete, there exist $p, q, r, u, v, w \in X$ such that $g x_{n} \rightarrow p=g u, g y_{n} \rightarrow q=g v$ and $g z_{n} \rightarrow r=g w$. Since $g x_{n} \neq g x_{n+1}$ or $g y_{n} \neq g y_{n+1}$ or $g z_{n} \neq g z_{n+1}$ for all $n$, it follows that $g x_{n} \neq g u$ or $g y_{n} \neq g v$ or $g z_{n} \neq g w$ for infinitely many $n$.

Hence $\max \left\{d\left(g x_{n}, g u\right), d\left(g y_{n}, g v\right), d\left(g z_{n}, g w\right)\right\}>0$ for infinitely many $n$.

Claim I: We will prove that

$$
\max \left\{\begin{array}{c}
d(g u, F(x, y, z)), \\
d(g v, F(y, z, x)), \\
d(g w, F(z, x, y))
\end{array}\right\} \leq \theta \max \left\{\begin{array}{c}
d(g u, g x), d(g v, g y), \\
d(g w, g z), d(g x, F(x, y, z)), \\
d(g y, F(y, z, x)), d(g z, F(z, x, y))
\end{array}\right\},
$$

for all $x, y, z \in X$ with $\max \{d(g u, g x), d(g v, g y), d(g w, g z)\}>0$.

Let $x, y, z \in X$ be such that $\max \{d(g u, g x), d(g v, g y), d(g w, g z)\}>0$. Since $g x_{n} \rightarrow g u, g y_{n} \rightarrow g v$ and $g z_{n} \rightarrow g w$, there exists a positive integer $n_{0}$ such that for $n \geq n_{0}$, we have

$$
\max \left\{\begin{array}{c}
d\left(g x_{n}, g u\right) \\
d\left(g y_{n}, g v\right), \\
d\left(g z_{n}, g w\right)
\end{array}\right\} \leq \frac{1}{3} \max \left\{\begin{array}{c}
d(g u, g x) \\
d(g v, g y) \\
d(g w, g z)
\end{array}\right\}
$$


Now, for $n \geq n_{0}$, using (2.4) we have

$$
\begin{aligned}
& \phi(\theta) \min \left\{\begin{array}{c}
d\left(g x_{n}, F\left(x_{n}, y_{n}, z_{n}\right)\right), \\
d\left(g y_{n}, F\left(y_{n}, z_{n}, x_{n}\right)\right), \\
d\left(g z_{n}, F\left(z_{n}, x_{n}, y_{n}\right)\right)
\end{array}\right\} \\
& \leq \max \left\{\begin{array}{c}
d\left(g x_{n}, g x_{n+1}\right) \\
d\left(g y_{n}, g y_{n+1}\right) \\
d\left(g z_{n}, g z_{n+1}\right)
\end{array}\right\} \\
& \leq \max \left\{\begin{array}{l}
d\left(g x_{n}, g u\right)+d\left(g u, g x_{n+1}\right) \\
d\left(g y_{n}, g v\right)+d\left(g v, g y_{n+1}\right) \\
d\left(g z_{n}, g w\right)+d\left(g w, g z_{n+1}\right)
\end{array}\right\} \\
& \leq \max \left\{\begin{array}{c}
d\left(g x_{n}, g u\right), d\left(g y_{n}, g v\right), \\
d\left(g z_{n}, g w\right)
\end{array}\right\}+\max \left\{\begin{array}{c}
d\left(g u, g x_{n+1}\right), d\left(g v, g y_{n+1}\right), \\
d\left(g w, g z_{n+1}\right)
\end{array}\right\} \\
& \leq \frac{2}{3} \max \left\{\begin{array}{c}
d(g u, g x), \\
d(g v, g y), \\
d(g w, g z)
\end{array}\right\}=\max \left\{\begin{array}{c}
d(g u, g x), \\
d(g v, g y), \\
d(g w, g z)
\end{array}\right\}-\frac{1}{3} \max \left\{\begin{array}{c}
d(g u, g x), \\
d(g v, g y), \\
d(g w, g z)
\end{array}\right\} \\
& \leq \max \left\{\begin{array}{c}
d(g u, g x), \\
d(g v, g y), \\
d(g w, g z)
\end{array}\right\}-\max \left\{\begin{array}{c}
d\left(g u, g x_{n}\right), \\
d\left(g v, g y_{n}\right) \\
d\left(g w, g z_{n}\right)
\end{array}\right\} \\
& \leq \max \left\{\begin{array}{l}
d(g u, g x)-d\left(g u, g x_{n}\right) \\
d(g v, g y)-d\left(g v, g y_{n}\right) \\
d(g w, g z)-d\left(g w, g z_{n}\right)
\end{array}\right\} \\
& \leq \max \left\{d\left(g x_{n}, g x\right), d\left(g y_{n}, g y\right), d\left(g z_{n}, g z\right)\right\} .
\end{aligned}
$$

From (2.2) and using triangular inequality, we have

$H\left(F\left(x_{n}, y_{n}, z_{n}\right), F(x, y, z)\right)$

$$
\begin{aligned}
& \leq \theta \max \left\{\begin{array}{c}
d\left(g x_{n}, g x\right), d\left(g y_{n}, g y\right), d\left(g z_{n}, g z\right), \\
d\left(g x_{n}, g x_{n+1}\right), d\left(g y_{n}, g y_{n+1}\right), d\left(g z_{n}, g z_{n+1}\right), \\
d(g x, F(x, y, z)), d(g y, F(y, z, x)), d(g z, F(z, x, y)), \\
\frac{1}{2} \max \left\{\begin{array}{c}
d\left(g x_{n}, F(x, y, z)\right), d\left(g y_{n}, F(y, z, x)\right), d\left(g z_{n}, F(z, x, y)\right), \\
d\left(g x, g x_{n+1}\right), d\left(g y, g y_{n+1}\right), d\left(g z, g z_{n+1}\right)
\end{array}\right\}
\end{array}\right\} \\
& \leq \theta \max \left\{\begin{array}{c}
d\left(g x_{n}, g x\right), d\left(g y_{n}, g y\right), d\left(g z_{n}, g z\right), \\
d\left(g x_{n}, g x_{n+1}\right), d\left(g y_{n}, g y_{n+1}\right), d\left(g z_{n}, g z_{n+1}\right), \\
d(g x, F(x, y, z)), d(g y, F(y, z, x)), d(g z, F(z, x, y))
\end{array}\right\} .
\end{aligned}
$$


Similarly, we can show that

$$
\begin{aligned}
& H\left(F\left(y_{n}, z_{n}, x_{n}\right), F(y, z, x)\right) \\
& \leq \theta \max \left\{\begin{array}{c}
d\left(g x_{n}, g x\right), d\left(g y_{n}, g y\right), d\left(g z_{n}, g z\right), \\
d\left(g x_{n}, g x_{n+1}\right), d\left(g y_{n}, g y_{n+1}\right), d\left(g z_{n}, g z_{n+1}\right), \\
d(g x, F(x, y, z)), d(g y, F(y, z, x)), d(g z, F(z, x, y))
\end{array}\right\},
\end{aligned}
$$

and

$$
\begin{aligned}
& H\left(F\left(z_{n}, x_{n}, y_{n}\right), F(z, x, y)\right) \\
& \leq \theta \max \left\{\begin{array}{c}
d\left(g x_{n}, g x\right), d\left(g y_{n}, g y\right), d\left(g z_{n}, g z\right), \\
d\left(g x_{n}, g x_{n+1}\right), d\left(g y_{n}, g y_{n+1}\right), d\left(g z_{n}, g z_{n+1}\right), \\
d(g x, F(x, y, z)), d(g y, F(y, z, x)), d(g z, F(z, x, y))
\end{array}\right\} .
\end{aligned}
$$

Thus, we get

$$
\begin{aligned}
& \max \left\{\begin{array}{c}
d\left(g x_{n+1}, F(x, y, z)\right), \\
d\left(g y_{n+1}, F(y, z, x)\right), \\
d\left(g z_{n+1}, F(z, x, y)\right)
\end{array}\right\} \\
& \leq \max \left\{\begin{array}{c}
H\left(F\left(x_{n}, y_{n}, z_{n}\right), F(x, y, z)\right), \\
H\left(F\left(y_{n}, z_{n}, x_{n}\right), F(y, z, x)\right), \\
H\left(F\left(z_{n}, x_{n}, y_{n}\right), F(z, x, y)\right)
\end{array}\right\} \\
& \leq \theta \max \left\{\begin{array}{c}
d\left(g x_{n}, g x_{n+1}\right), d\left(g y_{n}, g y_{n+1}\right), d\left(g z_{n}, g z_{n+1}\right), \\
d(g x, F(x, y, z)), d(g y, F(y, z, x)), d(g z, F(z, x, y))
\end{array}\right\} .
\end{aligned}
$$

Letting $n \rightarrow \infty$, we get

$$
\max \left\{\begin{array}{l}
d(g u, F(x, y, z)), \\
d(g v, F(y, z, x)), \\
d(g w, F(z, x, y))
\end{array}\right\} \leq \theta \max \left\{\begin{array}{c}
d(g u, g x), d(g v, g y), \\
d(g w, g z), d(g x, F(x, y, z)), \\
d(g y, F(y, z, x)), d(g z, F(z, x, y))
\end{array}\right\} .
$$

Hence the Claim I is true. Now we will show that $(u, v, w)$ is a tripled coincidence point of $F$ and $g$.

Case a: Suppose now that $0 \leq \theta<\frac{1}{2}$.

On the contrary, assume that $g u \notin F(u, v, w)$ or $g v \notin F(v, w, u)$ or $g w \notin F(w, u, v)$. Let $g a \in F(u, v, w), g b \in F(v, w, u)$ and $g c \in F(w, u, v)$ be such that

$$
2 \theta \max \left\{\begin{array}{c}
d(g a, g u), \\
d(g b, g v), \\
d(g c, g w)
\end{array}\right\}<\max \left\{\begin{array}{c}
d(g u, F(u, v, w)), \\
d(g v, F(v, w, u)), \\
d(g w, F(w, u, v)) .
\end{array}\right\}
$$


Since $g a \in F(u, v, w), g b \in F(v, w, u)$ and $g c \in F(w, u, v)$, we have $g a \neq g u$ or $g b \neq g v$ or $g c \neq g w$ and hence $a \neq u$ or $b \neq v$ or $c \neq w$. From Claim I, we have

$$
\max \left\{\begin{array}{c}
d(g u, F(a, b, c)), \\
d(g v, F(b, c, a)), \\
d(g w, F(c, a, b))
\end{array}\right\} \leq \theta \max \left\{\begin{array}{c}
d(g u, g a), d(g v, g b), \\
d(g w, g c), d(g a, F(a, b, c)), \\
d(g b, F(b, c, a)), d(g c, F(c, a, b))
\end{array}\right\} .
$$

Since

$$
\phi(\theta) \min \left\{\begin{array}{c}
d(g u, F(u, v, w)), \\
d(g v, F(v, w, u)), \\
d(g w, F(w, u, v))
\end{array}\right\} \leq \max \left\{\begin{array}{c}
d(g u, g a) \\
d(g v, g b) \\
d(g w, g c)
\end{array}\right\}
$$

from (2.2) and triangular inequality we have

$$
\begin{aligned}
& H(F(u, v, w), F(a, b, c)) \\
& \leq \theta \max \left\{\begin{array}{c}
d(g u, g a), d(g v, g b), d(g w, g c) \\
d(g u, F(u, v, w)), d(g v, F(v, w, u)), d(g w, F(w, u, v)) \\
d(g a, F(a, b, c)), d(g b, F(b, c, a)), d(g c, F(c, a, b)) \\
\frac{1}{2} \max \left\{\begin{array}{c}
d(g u, F(a, b, c)), d(g v, F(b, c, a)), d(g w, F(c, a, b)) \\
d(g a, F(u, v, w)), d(g b, F(v, w, u)), d(g c, F(w, u, v))
\end{array}\right\}
\end{array}\right\} \\
& \leq \theta \max \left\{\begin{array}{c}
d(g u, g a), d(g v, g b), d(g w, g c), \\
d(g a, F(a, b, c)), d(g b, F(b, c, a)), d(g c, F(c, a, b))
\end{array}\right\}
\end{aligned}
$$

Similarly, we have

$$
H(F(v, w, u), F(b, c, a)) \leq \theta \max \left\{\begin{array}{c}
d(g u, g a), d(g v, g b), \\
d(g w, g c), d(g a, F(a, b, c)), \\
d(g b, F(b, c, a)), d(g c, F(c, a, b))
\end{array}\right\},
$$

and

$$
H(F(w, u, v), F(c, a, b)) \leq \theta \max \left\{\begin{array}{c}
d(g u, g a), d(g v, g b), \\
d(g w, g c), d(g a, F(a, b, c)), \\
d(g b, F(b, c, a)), d(g c, F(c, a, b))
\end{array}\right\} .
$$

Thus

$$
\max \left\{\begin{array}{c}
H(F(u, v, w), F(a, b, c)), \\
H(F(v, w, u), F(b, c, a)), \\
H(F(w, u, v), F(c, a, b))
\end{array}\right\} \leq \theta \max \left\{\begin{array}{c}
d(g u, g a), d(g v, g b), \\
d(g w, g c), d(g a, F(a, b, c)), \\
d(g b, F(b, c, a)), d(g c, F(c, a, b))
\end{array}\right\} .
$$

From (2.7) we have

$$
\max \left\{\begin{array}{c}
d(g a, F(a, b, c)), \\
d(g b, F(b, c, a)), \\
d(g c, F(c, a, b))
\end{array}\right\} \leq \max \left\{\begin{array}{c}
H(F(u, v, w), F(a, b, c)) \\
H(F(v, w, u), F(b, c, a)), \\
H(F(w, u, v), F(c, a, b))
\end{array}\right\}
$$




$$
\leq \theta \max \left\{\begin{array}{c}
d(g u, g a), d(g v, g b) \\
d(g w, g c), d(g a, F(a, b, c)) \\
d(g b, F(b, c, a)), d(g c, F(c, a, b))
\end{array}\right\}
$$

If

$$
\max \left\{\begin{array}{c}
d(g u, g a), d(g v, g b), \\
d(g w, g c), d(g a, F(a, b, c)), \\
d(g b, F(b, c, a)), d(g c, F(c, a, b))
\end{array}\right\}=d(g a, F(a, b, c))
$$

we conclude that $g a \in F(a, b, c)$. Analogous we conclude that $g b \in F(b, c, a)$ and $g c \in F(c, a, b)$ if

$$
\max \left\{\begin{array}{c}
d(g u, g a), d(g v, g b), \\
d(g w, g c), d(g a, F(a, b, c)), \\
d(g b, F(b, c, a)), d(g c, F(c, a, b))
\end{array}\right\}=d(g b, F(b, c, a))
$$

or

$$
\max \left\{\begin{array}{c}
d(g u, g a), d(g v, g b), \\
d(g w, g c), d(g a, F(a, b, c)), \\
d(g b, F(b, c, a)), d(g c, F(c, a, b))
\end{array}\right\}=d(g c, F(c, a, b)) .
$$

This is contradiction with $a \neq u, b \neq v$ and $c \neq w$.

So,

$$
\max \left\{\begin{array}{c}
d(g a, F(a, b, c)), \\
d(g b, F(b, c, a)), \\
d(g c, F(c, a, b))
\end{array}\right\} \leq \theta \max \left\{\begin{array}{c}
d(g u, g a) \\
d(g v, g b), \\
d(g w, g c)
\end{array}\right\}
$$

From (2.6) and (2.8) we have

$$
\max \left\{\begin{array}{c}
d(g u, F(a, b, c)) \\
d(g v, F(b, c, a)), \\
d(g w, F(c, a, b))
\end{array}\right\} \leq \theta \max \left\{\begin{array}{c}
d(g u, g a) \\
d(g v, g b), \\
d(g w, g c)
\end{array}\right\}
$$

and from (2.7) we get

$$
\max \left\{\begin{array}{c}
H(F(u, v, w), F(a, b, c)), \\
H(F(v, w, u), F(b, c, a)), \\
H(F(w, u, v), F(c, a, b))
\end{array}\right\} \leq \theta \max \left\{\begin{array}{c}
d(g u, g a), \\
d(g v, g b), \\
d(g w, g c)
\end{array}\right\} .
$$


Now, using (2.5), (2.9) and (2.10) we obtain

$$
\begin{aligned}
& \max \left\{\begin{array}{l}
d(g u, F(u, v, w)), \\
d(g v, F(v, w, u)), \\
d(g w, F(w, u, v))
\end{array}\right\} \\
& \leq \max \left\{\begin{array}{c}
d(g u, F(a, b, c))+H(F(a, b, c), F(u, v, w)) \\
d(g v, F(b, c, a))+H(F(b, c, a), F(v, w, u)) \\
d(g w, F(c, a, b))+H(F(c, a, b), F(w, u, v))
\end{array}\right\} \\
& \leq \max \left\{\begin{array}{c}
d(g u, F(a, b, c)), \\
d(g v, F(b, c, a)), \\
d(g w, F(c, a, b))
\end{array}\right\}+\max \left\{\begin{array}{c}
H(F(a, b, c), F(u, v, w)), \\
H(F(b, c, a), F(v, w, u)), \\
H(F(c, a, b), F(w, u, v))
\end{array}\right\} \\
& \leq 2 \theta \max \left\{\begin{array}{c}
d(g u, g a), \\
d(g v, g b), \\
d(g w, g c)
\end{array}\right\}<\max \left\{\begin{array}{c}
d(g u, F(u, v, w)), \\
d(g v, F(v, w, u)), \\
d(g w, F(w, u, v))
\end{array}\right\} .
\end{aligned}
$$

It is a contradiction. Hence $g u \in F(u, v, w), g v \in F(v, w, u)$ and $g w \in F(w, u, v)$.

Case b: Suppose now that $\frac{1}{2} \leq \theta<1$.

Claim II: We will show that

$$
\begin{gathered}
\max \left\{\begin{array}{c}
H(F(x, y, z), F(u, v, w)), \\
H(F(y, z, x), F(v, w, u)), \\
H(F(z, x, y), F(w, u, v))
\end{array}\right\} \\
\leq \theta \max \left\{\begin{array}{c}
d(g x, g u), d(g y, g v), d(g z, g w), \\
d(g x, F(x, y, z)), d(g y, F(y, z, x)), d(g z, F(z, x, y)), \\
d(g u, F(u, v, w)), d(g v, F(v, w, u)), d(g w, F(w, u, v)), \\
\frac{1}{2} \max \left\{\begin{array}{c}
d(g x, F(u, v, w)), d(g y, F(v, w, u)), d(g z, F(w, u, v)), \\
d(g u, F(x, y, z)), d(g v, F(y, z, x)), d(g w, F(z, x, y))
\end{array}\right\}
\end{array}\right\},
\end{gathered}
$$

for all $x, y, z \in X$ with $\max \{d(g x, g u), d(g y, g v), d(g z, g w)\}>0$.

Let $x, y, z \in X$ be such that $\max \{d(g x, g u), d(g y, g v), d(g z, g w)\}>0$. Then for every positive integer $n$, there exist sequences $t_{n} \in F(x, y, z), t_{n}^{1} \in F(y, z, x)$ and $t_{n}^{11} \in F(z, x, y)$ such that

$$
\max \left\{\begin{array}{c}
d\left(g u, t_{n}\right), \\
d\left(g v, t_{n}^{1}\right), \\
d\left(g w, t_{n}^{11}\right)
\end{array}\right\} \leq \max \left\{\begin{array}{c}
d(g u, F(x, y, z)), \\
d(g v, F(y, z, x)), \\
d(g w, F(z, x, y))
\end{array}\right\}+\frac{1}{n} \max \left\{\begin{array}{c}
d(g x, g u), \\
d(g y, g v), \\
d(g z, g w)
\end{array}\right\} .
$$


Now, using (2.11) and triangular inequality we obtain

$$
\begin{gathered}
\max \left\{\begin{array}{c}
d(g x, F(x, y, z)), \\
d(g y, F(y, z, x)), \\
d(g z, F(z, x, y))
\end{array}\right\} \leq \max \left\{\begin{array}{c}
d(g x, g u)+d\left(g u, t_{n}\right), \\
d(g y, g v)+d\left(g v, t_{n}^{1}\right), \\
d(g z, g w)+d\left(g w, t_{n}^{11}\right)
\end{array}\right\} \\
\leq \max \left\{\begin{array}{c}
d(g x, g u), \\
d(g y, g v), \\
d(g z, g w)
\end{array}\right\}+\max \left\{\begin{array}{c}
d\left(g u, t_{n}\right), \\
d\left(g v, t_{n}^{1}\right), \\
d\left(g w, t_{n}^{11}\right)
\end{array}\right\} \\
\leq \max \left\{\begin{array}{c}
d(g x, g u), \\
d(g y, g v), \\
d(g z, g w)
\end{array}\right\}+\max \left\{\begin{array}{c}
d(g u, F(x, y, z)), \\
d(g v, F(y, z, x)), \\
d(g w, F(z, x, y))
\end{array}\right\}+\frac{1}{n} \max \left\{\begin{array}{c}
d(g x, g u), \\
d(g y, g v), \\
d(g z, g w)
\end{array}\right\} \\
\leq\left(1+\frac{1}{n}\right) \max \left\{\begin{array}{c}
d(g x, g u), \\
d(g y, g v), \\
d(g z, g w)
\end{array}\right\}+\theta \max \left\{\begin{array}{c}
d(g u, g x), d(g v, g y), \\
d(g w, g z), d(g x, F(x, y, z)), \\
d(g y, F(y, z, x)), d(g z, F(z, x, y))
\end{array}\right\} .
\end{gathered}
$$

If

$$
\max \left\{\begin{array}{l}
d(g u, g x), \\
d(g v, g y), \\
d(g w, g z)
\end{array}\right\} \geq \max \left\{\begin{array}{c}
d(g x, F(x, y, z)), \\
d(g y, F(y, z, x)), \\
d(g z, F(z, x, y))
\end{array}\right\},
$$

then from (2.12) we have

$$
\max \left\{\begin{array}{c}
d(g x, F(x, y, z)), \\
d(g y, F(y, z, x)), \\
d(g z, F(z, x, y))
\end{array}\right\} \leq\left(1+\theta+\frac{1}{n}\right) \max \left\{\begin{array}{c}
d(g u, g x), \\
d(g v, g y), \\
d(g w, g z)
\end{array}\right\} .
$$

Letting $n \rightarrow \infty$, we get

$$
\max \left\{\begin{array}{c}
d(g x, F(x, y, z)), \\
d(g y, F(y, z, x)), \\
d(g z, F(z, x, y))
\end{array}\right\} \leq(1+\theta) \max \left\{\begin{array}{c}
d(g u, g x), \\
d(g v, g y), \\
d(g w, g z)
\end{array}\right\} .
$$

By (1.1), as $\frac{1}{2} \leq \theta<1$ we have $\phi(\theta)=1-\theta$, using (2.13) we have

$$
\begin{aligned}
& \phi(\theta) \min \left\{\begin{array}{c}
d(g x, F(x, y, z)), \\
d(g y, F(y, z, x)), \\
d(g z, F(z, x, y))
\end{array}\right\} \leq(1-\theta) \max \left\{\begin{array}{c}
d(g x, F(x, y, z)), \\
d(g y, F(y, z, x)), \\
d(g z, F(z, x, y))
\end{array}\right\} \\
& \leq\left(1-\theta^{2}\right) \max \left\{\begin{array}{c}
d(g u, g x), \\
d(g v, g y), \\
d(g w, g z)
\end{array}\right\}<\max \left\{\begin{array}{c}
d(g u, g x), \\
d(g v, g y), \\
d(g w, g z)
\end{array}\right\} .
\end{aligned}
$$

If

$$
\max \left\{\begin{array}{l}
d(g u, g x), \\
d(g v, g y), \\
d(g w, g z)
\end{array}\right\}<\max \left\{\begin{array}{c}
d(g x, F(x, y, z)) \\
d(g y, F(y, z, x)) \\
d(g z, F(z, x, y))
\end{array}\right\}
$$


then from (2.12), we have

$$
(1-\theta) \max \left\{\begin{array}{c}
d(g x, F(x, y, z)), \\
d(g y, F(y, z, x)), \\
d(g z, F(z, x, y))
\end{array}\right\} \leq\left(1+\frac{1}{n}\right) \max \left\{\begin{array}{c}
d(g u, g x), \\
d(g v, g y), \\
d(g w, g z)
\end{array}\right\} .
$$

Letting $n \rightarrow \infty$, we get

$$
\begin{gathered}
\phi(\theta) \min \left\{\begin{array}{c}
d(g x, F(x, y, z)), \\
d(g y, F(y, z, x)), \\
d(g z, F(z, x, y))
\end{array}\right\} \leq(1-\theta) \max \left\{\begin{array}{c}
d(g x, F(x, y, z)), \\
d(g y, F(y, z, x)), \\
d(g z, F(z, x, y))
\end{array}\right\} \\
\leq \max \left\{\begin{array}{c}
d(g u, g x), \\
d(g v, g y), \\
d(g w, g z)
\end{array}\right\} .
\end{gathered}
$$

Thus, in both cases we have

$$
\phi(\theta) \min \left\{\begin{array}{c}
d(g x, F(x, y, z)), \\
d(g y, F(y, z, x)), \\
d(g z, F(z, x, y))
\end{array}\right\} \leq \max \left\{\begin{array}{c}
d(g u, g x), \\
d(g v, g y), \\
d(g w, g z)
\end{array}\right\} .
$$

From (2.2) and (2.15), we have

$$
H(F(x, y, z), F(u, v, w))
$$

$$
\leq \theta \max \left\{\begin{array}{c}
d(g x, g u), d(g y, g v), d(g z, g w), \\
d(g x, F(x, y, z)), d(g y, F(y, z, x)), d(g z, F(z, x, y)), \\
d(g u, F(u, v, w)), d(g v, F(v, w, u)), d(g w, F(w, u, v)), \\
\frac{1}{2} \max \left\{\begin{array}{c}
d(g x, F(u, v, w)), d(g y, F(v, w, u)), d(g z, F(w, u, v)), \\
d(g u, F(x, y, z)), d(g v, F(y, z, x)), d(g w, F(z, x, y))
\end{array}\right\}
\end{array}\right\} .
$$

Similarly, we can show that

$$
H(F(y, z, x), F(v, w, u))
$$$$
\leq \theta \max \left\{\begin{array}{c}
d(g x, g u), d(g y, g v), d(g z, g w), \\
d(g x, F(x, y, z)), d(g y, F(y, z, x)), d(g z, F(z, x, y)), \\
d(g u, F(u, v, w)), d(g v, F(v, w, u)), d(g w, F(w, u, v)), \\
\frac{1}{2} \max \left\{\begin{array}{c}
d(g x, F(u, v, w)), d(g y, F(v, w, u)), d(g z, F(w, u, v)), \\
d(g u, F(x, y, z)), d(g v, F(y, z, x)), d(g w, F(z, x, y))
\end{array}\right\}
\end{array}\right\},
$$ 
and

$H(F(z, x, y), F(w, u, v))$

$$
\leq \theta \max \left\{\begin{array}{c}
d(g x, g u), d(g y, g v), d(g z, g w), \\
d(g x, F(x, y, z)), d(g y, F(y, z, x)), d(g z, F(z, x, y)), \\
d(g u, F(u, v, w)), d(g v, F(v, w, u)), d(g w, F(w, u, v)), \\
\frac{1}{2} \max \left\{\begin{array}{c}
d(g x, F(u, v, w)), d(g y, F(v, w, u)), d(g z, F(w, u, v)), \\
d(g u, F(x, y, z)), d(g v, F(y, z, x)), d(g w, F(z, x, y))
\end{array}\right\}
\end{array}\right\} .
$$

Thus

$$
\begin{aligned}
& \max \left\{\begin{array}{c}
H(F(x, y, z), F(u, v, w)), \\
H(F(y, z, x), F(v, w, u)), \\
H(F(z, x, y), F(w, u, v))
\end{array}\right\} \\
& \leq \theta \max \left\{\begin{array}{c}
d(g x, g u), d(g y, g v), d(g z, g w), \\
d(g x, F(x, y, z)), d(g y, F(y, z, x)), d(g z, F(z, x, y)), \\
d(g u, F(u, v, w)), d(g v, F(v, w, u)), d(g w, F(w, u, v)), \\
\frac{1}{2} \max \left\{\begin{array}{c}
d(g x, F(u, v, w)), d(g y, F(v, w, u)), d(g z, F(w, u, v)), \\
d(g u, F(x, y, z)), d(g v, F(y, z, x)), d(g w, F(z, x, y))
\end{array}\right\}
\end{array}\right\} .
\end{aligned}
$$

Hence the Claim II is true. Now consider

$$
\begin{aligned}
& \max \left\{\begin{array}{c}
d(g u, F(u, v, w)), \\
d(g v, F(v, w, u)), \\
d(g w, F(w, u, v))
\end{array}\right\} \\
& \leq \lim _{n \rightarrow \infty} \max \left\{\begin{array}{c}
d\left(g x_{n+1}, F(u, v, w)\right), \\
d\left(g y_{n+1}, F(v, w, u)\right), \\
d\left(g z_{n+1}, F(w, u, v)\right)
\end{array}\right\} \\
& \leq \lim _{n \rightarrow \infty} \max \left\{\begin{array}{c}
H\left(F\left(x_{n}, y_{n}, z_{n}\right), F(u, v, w)\right), \\
H\left(F\left(y_{n}, z_{n}, x_{n}\right), F(v, w, u)\right), \\
H\left(F\left(z_{n}, x_{n}, y_{n}\right), F(w, u, v)\right)
\end{array}\right\} \\
& \leq \lim _{n \rightarrow \infty} \theta \max \left\{\begin{array}{c}
d\left(g x_{n}, g u\right), d\left(g y_{n}, g v\right), d\left(g z_{n}, g w\right), \\
d\left(g x_{n}, F\left(x_{n}, y_{n}, z_{n}\right)\right), d\left(g y_{n}, F\left(y_{n}, z_{n}, x_{n}\right)\right), \\
d\left(g z_{n}, F\left(z_{n}, x_{n}, y_{n}\right)\right), d(g u, F(u, v, w)), \\
d(g v, F(v, w, u)), d(g w, F(w, u, v)), \\
d\left(g x_{n}, F(u, v, w)\right), d\left(g y_{n}, F(v, w, u)\right), \\
d\left(g z_{n}, F(w, u, v)\right), d\left(g u, F\left(x_{n}, y_{n}, z_{n}\right)\right), \\
d\left(g v, F\left(y_{n}, z_{n}, x_{n}\right)\right), d\left(g w, F\left(z_{n}, x_{n}, y_{n}\right)\right)
\end{array}\right\}
\end{aligned}
$$


$\leq \theta \lim _{n \rightarrow \infty} \max \left\{\begin{array}{c}d\left(g x_{n}, g u\right), d\left(g y_{n}, g v\right), d\left(g z_{n}, g w\right), \\ d\left(g x_{n}, g x_{n+1}\right), d\left(g y_{n}, g y_{n+1}\right), d\left(g z_{n}, g z_{n+1}\right), \\ d(g u, F(u, v, w)), d(g v, F(v, w, u)), d(g w, F(w, u, v)), \\ \max \left\{\begin{array}{c}d\left(g x_{n}, F(u, v, w)\right), d\left(g y_{n}, F(v, w, u)\right), d\left(g z_{n}, F(w, u, v)\right), \\ d\left(g u, g x_{n+1}\right), d\left(g v, g y_{n+1}\right), d\left(g w, g z_{n+1}\right)\end{array}\right\}\end{array}\right\}$.

Letting $n \rightarrow \infty$ we get

$$
\max \left\{\begin{array}{l}
d(g u, F(u, v, w)) \\
d(g v, F(v, w, u)) \\
d(g w, F(w, u, v))
\end{array}\right\} \leq \theta \max \left\{\begin{array}{c}
d(g u, F(u, v, w)) \\
d(g v, F(v, w, u)) \\
d(g w, F(w, u, v))
\end{array}\right\}
$$

which in turn yields that $g u \in F(u, v, w), g v \in F(v, w, u)$ and $g w \in F(w, u, v)$. Thus $(u, v, w)$ is a tripled coincidence point of $F$ and $g$.

Suppose now that (a) holds. Let $(x, y, z)$ be a tripled coincidence point of $F$ and $g$. Then there exist $u, v, w \in X$ such that $\lim _{n \rightarrow \infty} g^{n} x=u, \lim _{n \rightarrow \infty} g^{n} y=v$ and $\lim _{n \rightarrow \infty} g^{n} z=w$.

Since $g$ is continuous at $u, v, w$, we have $g u=u, g v=v$ and $g w=w$. Based on the assumption that the pair $(F, g)$ is $w$-compatible and $g x \in F(x, y, z), g y \in$ $F(y, z, x)$ and $g z \in F(z, x, y)$, we have $g^{2} x \in F(g x, g y, g z), g^{2} y \in F(g y, g z, g x)$ and $g^{2} z \in F(g z, g x, g y)$ respectively. Thus $(g x, g y, g z)$ is a tripled coincidence point of $F$ and $g$. Similarly, we can show that $\left(g^{n} x, g^{n} y, g^{n} z\right)$ is a tripled coincidence point of $F$ and $g$. Also it is clear that $g^{n} x \in F\left(g^{n-1} x, g^{n-1} y, g^{n-1} z\right)$, $g^{n} y \in F\left(g^{n-1} y, g^{n-1} z, g^{n-1} x\right)$ and $g^{n} z \in F\left(g^{n-1} z, g^{n-1} x, g^{n-1} y\right)$. Now we have $\phi(\theta) \min \left\{\begin{array}{l}d\left(g^{n} x, F\left(g^{n-1} x, g^{n-1} y, g^{n-1} z\right)\right), \\ d\left(g^{n} y, F\left(g^{n-1} y, g^{n-1} z, g^{n-1} x\right)\right), \\ d\left(g^{n} z, F\left(, g^{n-1} z, g^{n-1} x, g^{n-1} y\right)\right)\end{array}\right\}=0 \leq \max \left\{\begin{array}{c}d\left(g^{n} x, g u\right), \\ d\left(g^{n} y, g v\right), \\ d\left(g^{n} z, g w\right),\end{array}\right\}$.

Hence from (2.2), we have

$$
\begin{aligned}
& \left.d\left(g^{n} x, F(u, v, w)\right)\right) \leq H\left(F\left(g^{n-1} x, g^{n-1} y, g^{n-1} z\right), F(u, v, w)\right) \\
& \leq \theta \max \left\{\begin{array}{c}
d\left(g^{n} x, g u\right), d\left(g^{n} y, g v\right), d\left(g^{n} z, g w\right) \\
d\left(g^{n} x, F\left(g^{n-1} x, g^{n-1} y, g^{n-1} z\right)\right), d\left(g^{n} y, F\left(g^{n-1} y, g^{n-1} z, g^{n-1} x\right)\right) \\
d\left(g^{n} z, F\left(g^{n-1} z, g^{n-1} x, g^{n-1} y\right)\right), d(g u, F(u, v, w)), \\
d(g v, F(v, w, u)), d(g w, F(w, u, v)) \\
d\left(g^{n} x, F(u, v, w)\right), d\left(g^{n} y, F(v, w, u)\right), \\
\frac{1}{2} \max \left\{\begin{array}{c}
d\left(g^{n} z, F(w, u, v)\right), d\left(g u, F\left(g^{n-1} x, g^{n-1} y, g^{n-1} z\right)\right), \\
d\left(g v, F\left(g^{n-1} y, g^{n-1} z, g^{n-1} x\right)\right), d\left(g w, F\left(g^{n-1} z, g^{n-1} x, g^{n-1} y\right)\right)
\end{array}\right\}
\end{array}\right\} .
\end{aligned}
$$

Letting $n \rightarrow \infty$, we get

$$
d(u, F(u, v, w)) \leq \theta \max \{d(u, F(u, v, w)), d(v, F(v, w, u)), d(w, F(w, u, v))\} .
$$

Similarly, we have

$$
d(v, F(v, w, u)) \leq \theta \max \{d(u, F(u, v, w)), d(v, F(v, w, u)), d(w, F(w, u, v))\},
$$


and

$$
d(w, F(w, u, v)) \leq \theta \max \{d(u, F(u, v, w)), d(v, F(v, w, u)), d(w, F(w, u, v))\} .
$$

Thus

$$
\max \left\{\begin{array}{l}
d(u, F(u, v, w)), \\
d(v, F(v, w, u)), \\
d(w, F(w, u, v))
\end{array}\right\} \leq \theta \max \left\{\begin{array}{c}
d(u, F(u, v, w)), \\
d(v, F(v, w, u)), \\
d(w, F(w, u, v))
\end{array}\right\}
$$

which in turn yields that $u \in F(u, v, w), v \in F(v, w, u)$ and $w \in F(w, u, v)$. Thus $(u, v, w)$ is a tripled common fixed point of $F$ and $g$.

Suppose now that (b) holds. Let $(x, y, z)$ be a tripled coincidence point of $F$ and $g$. Then there exist $u, v, w \in X$ such that $\lim _{n \rightarrow \infty} g^{n} u=x, \lim _{n \rightarrow \infty} g^{n} v=y$ and $\lim _{n \rightarrow \infty} g^{n} w=z$. Since $g$ is continuous at $x, y$ and $z$, we have $g x=x, g y=y$ and $g z=z$. Thus $(x, y, z)$ is a tripled common fixed point of $F$ and $g$.

The following example illustrates Theorem 3.

Example 1. Let $X=[0,1], F: X \times X \times X \rightarrow C B(X)$ and $g: X \rightarrow X$ be defined by $F(x, y, z)=\left[0, \frac{x}{8}+\frac{y}{4}+\frac{z}{3}\right]$ and $g x=\frac{7 x}{8}$. Then for all $x, y, z, u, v, w \in X$, consider

$$
\begin{aligned}
H(F(x, y, z), F(u, v, w)) & =\left|\left(\frac{x}{8}+\frac{y}{4}+\frac{z}{3}\right)-\left(\frac{u}{8}+\frac{v}{4}+\frac{w}{3}\right)\right| \\
& \leq \frac{1}{8}|x-u|+\frac{1}{4}|y-v|+\frac{1}{3}|z-w| \\
& =\frac{1}{7}|g x-g u|+\frac{2}{7}|g y-g v|+\frac{8}{21}|g z-g w| \\
& \leq \frac{17}{21} \max \{d(g x, g u), d(g y, g v), d(g z, g w)\} .
\end{aligned}
$$

Clearly all conditions of Theorem 3 are satisfied and $(0,0,0)$ is the tripled common fixed point of $F$ and $g$.

\section{REFERENCES}

[1] M. Abbas, H. Aydi, and E. Karapinar, "Tripled fixed points of multi valued nonlinear contraction mappings in partially ordered metric spaces," Abstract Applied Analysis, vol. 2012, p. Article ID 812690, 2012, doi: 10.1155/2011/812690.

[2] M. Aggarwal, R. Chugh, and R. Kamal, "Suzuki type fixed point results in g-metric spaces and applications," International J. Computer Appl., vol. 47, no. 12, pp. 14-17, 2012.

[3] I. Altun, "A common fixed point theorem for multi - valued Ćirić type mappings with new type compatibility," An. St. Univ. Ovidius Constanta, vol. 17, no. 2, pp. 19-26, 2009.

[4] I. Altun and A. Erduran, "A Suzuki type fixed point theorem," Internat. Math. Math. Sci., vol. 2011, no. Article ID 736063, p. 9 pages, 2011, doi: 10.1155/2011/736063. 
[5] H. Aydi, M. Abbas, W. Sintunavarat, and P. Kumam, "Tripled fixed point of w-compatible mappings in abstract metric spaces," Fixed Point Theory Appl., vol. 2012, no. 2012:134, 2012, doi: 10.1186/1687-1812-2012-134.

[6] H. Aydi and E. Karapinar, "New Meier-Keeler type tripled fixed point theorems on ordered partial metric spaces," Math. Probl. Eng., vol. 2012, no. Article ID 409872, 2012, doi: $10.1155 / 2012 / 409872$.

[7] H. Aydi, E. Karapinar, and M. Postolache, "Tripled coincidence point theorems for weak $\phi$ contractions in partially ordered metric spaces," Fixed Point Theory Appl., vol. 2012, no. 2012:44, 2012, doi: 10.1186/1687-1812-2012-44.

[8] H. Aydi, E. Karapinar, and W. Shatanawi, "Tripled coincidence point results for generalized contractions in ordered generalized metric spaces," Fixed Point Theory Appl., vol. 2012, no. 2012:101, 2012, doi: 10.1186/1687-1812-2012-101.

[9] H. Aydi, E. Karapinar, and W. Shatanawi, "Tripled fixed point results in generalized metric spaces," J. Applied Math., vol. 2012, no. Article ID 314279, p. 10 pages, 2012, doi: $10.1155 / 2012 / 314279$.

[10] S. Banach, "Sur les operations dans les ensembles abstraits et leur applications aux equations integrales," Fund. Math., vol. 3, no. 1, pp. 133-181, 1922.

[11] V. Berinde and M. Borcut, "Tripled fixed point theorems for contractive type mappings in partially ordered metric spaces," Nonlinear Anal., TMA., vol. 74, no. 15, pp. 4889-4897, 2011, doi: 10.1016/j.na.2011.03.032.

[12] V. Berinde and M. Borcut, "Tripled coincidence theorems for contractive type mappings in partially ordered metric spaces," Applied Math. Comput., vol. 218, pp. 7339-7346, 2012, doi: 10.1016/j.amc.2012.01.030.

[13] R. Bose and M. R. Chowdhury, "Fixed point theorems for some generalized contractive multi valued mappings and fuzzy mappings," Mat. Vesnik, vol. 63, no. 1, pp. 7-26, 2011.

[14] B. Choudhury, E. Karapinar, and A. Kundu, "Tripled coincidence point theorems for nonlinear contractions in partially ordered metric spaces," Internat. J. Math. Math. Sci., vol. 2012, no. Article ID 329298., p. 14 pages, 2012, doi: 10.1155/2012/329298.

[15] R. Chugh, R. Kamal, and M. Aggarwal, "Properties $P$ and $Q$ for Suzuki type fixed point theorems in metric spaces," International Journal of Computer Appl., vol. 50, no. 1, pp. 44-48, 2012.

[16] L. Ćirić, "Fixed point theorems for multi - valued contractions in complete metric spaces," $J$. Math. Anal. Appl., vol. 348, pp. 499 - 507, 2008, doi: 10.1016/j.jmaa.2008.07.062.

[17] L. Ćirić, "Multi - valued nonlinear contraction mappings," Nonlinear Anal., TMA., vol. 71, pp. 2716 - 2723, 2009, doi: 10.1016/j.na.2009.01.116.

[18] L. Ćirić and J. Ume, "Common fixed point theorems for multi - valued non - self mappings," Publ. Math. Debrecen, vol. 60, pp. 359 - 371, 2002.

[19] D. Djorić, Z. Kadelburg, and S. Radenović, "Edelstein-Suzuki type fixed point results in metric and abstract metric spaces," Nonlinear Anal. TMA., vol. 75, pp. 1927-1932, 2012, doi: 10.1016/j.na.2011.09.046.

[20] D. Djorić and R. Lazović, "Some Suzuki-type fixed point theorems for generalized multivalued mappings and applications," Fixed point Theory Appl., vol. 40, 2011, doi: 10.1186/1687-18122011-40.

[21] W. S. Du, "Some generalizations of Mizoguchi - Takahashi's fixed point theorem," Int. J. Contemp. Math. Sci., vol. 3, pp. 1283 - 1288, 2008.

[22] N. Hussain and A. Alotaibi, "Coupled coincidences for multi-valued contractions in partially ordered metric spaces," Fixed Point Theory Appl., vol. 2011, no. Article 82, 2011, doi: 10.1186/1687-1812-2011-82.

[23] N. Hussain, D. Djorić, Z. Kadelburg, and S. Radenović, "Suzuki type fixed point results in metric type spaces," Fixed Point Theory Appl., vol. 2012:126, 2012, doi: 10.1186/1687-1812-2012-126. 
[24] G. Jungck and B. E. Rhoades, "Fixed point for set valued functions without continuity," Indian. J. Pure Appl. Math., vol. 29, no. 3, pp. 227 - 238, 1998.

[25] E. Karapinar, "Edelstein type fixed point theorems," Ann. Funct. Anal., vol. 2, no. 1, pp. 51-58, 2011.

[26] H. Li, "s-coincidence and $s$-common fixed point theorems for two pairs of set-valued non- ompatible mappings in metric space," J. Nonlinear Sci. Appl., vol. 3, pp. 55-62, 2010.

[27] P. Murthy and Rashmi, "Tripled common fixed point theorems for w-compatible mappings in ordered cone metric spaces," Advances in Fixed Point Theory, vol. 2, no. 2, pp. 157-175, 2012.

[28] S. B. J. Nadler, "Multi - valued contraction mappings," Pacific J. Math., vol. 30, no. 2, pp. 475488, 1969.

[29] D. Paesano and P. Vetro, "Suzuki's type characterizations of completeness for partial metric spaces and fixed points for partially ordered metric spaces," Topology Appl., vol. 75, pp. 911-920, 2012, doi: 10.1016/j.topol.2011.12.008.

[30] K. P. R. Rao and G. N. V. Kishore, "A unique common tripled fixed point theorem in partially ordered cone metric spaces," Bulletin of Mathematical Analysis and Applications, vol. 3, no. 4, pp. 213-222, 2011.

[31] K. P. R. Rao, G. N. V. Kishore, and N. Srinivasa Rao, "A unique common 3-tupled fixed point theorem for $\psi-\phi$ contractions in partial metric spaces," Mathematica Aeterna, vol. 1, no. 7, pp. 491-507, 2011.

[32] K. P. R. Rao, G. N. V. Kishore, and K. Tas, "A unique common tripled fixed point theorem for hybrid pair of maps," Abstract Applied Analysis, vol. 2012, no. Article ID 750403, p. 9 pages, 2012, doi: 10.1155/2012/750403.

[33] B. Rhoades, "A fixed point theorem for a multi - valued non- self mapping," Comment. Math. Univ. Carolin., vol. 37, pp. 401-404, 1996.

[34] B. Samet and C. Vetro, "Coupled fixed point theorems for multi-valued nonlinear contraction mappings in partially ordered metric spaces," Nonlinear Anal. TMA., vol. 74, no. 12, pp. 4260 4268, 2011, doi: 10.1016/j.na.2011.04.007.

[35] S. Singh, S. Mishra, R. Chugh, and R. Kamal, "General common fixed point theorems and applications," J. Applied Math., vol. 2012, no. Article ID 902312, p. 14 pages, 2012, doi: 10.1155/2012/902312.

[36] S. Singh, H. Pathak, and S. Mishra, "On a Suzuki type general fixed point theorem with applications," Fixed point Theory Appl., vol. 2010, no. Article ID 234717, p. 15 pages, 2010, doi: 10.1155/2010/234717.

[37] T. Suzuki, "A generalized Banach contraction principle that characterizes metric completeness," Proceedings of the American Mathematical Society, vol. 136, no. 5, pp. 1861-1869, 2008, doi: 10.1090/S0002-9939-07-09055-7.

[38] T. Suzuki, "A new type of fixed point theorem in metric spaces," Nonlinear Anal., TMA., vol. 71, pp. 5313-5317, 2009, doi: 10.1016/j.na.2009.04.017.

\section{Authors' addresses}

\section{Stojan Radenović}

Nonlinear Analysis Research Group, Ton Duc Thang University, Ho Chi Minh City, Vietnam

Faculty of Mathematics and Statistics, Ton Duc Thang University, Ho Chi Minh City, Vietnam E-mail address: stojan.radenovic@tdt.edu.vn 
K. P. R. Rao

Department of Mathematics, Acharya Nagarjuna University, Nagarjuna Nagar-522 510, A. P., India E-mail address: kprrao2004@yahoo.com

K. V. Siva Parvathi

Department of Applied Mathematics, Krishna University-M.R.Appa Row P.G.Center, Nuzvid-521 201, Andhra Pradesh, India

E-mail address: kvsp1979@yahoo.com

Tatjana Došenović

Faculty of Technology, University of Novi Sad, Bulevar cara Lazara 1, Serbia

E-mail address: tat janadetf.uns.ac.rs 